\title{
Simuladores de formación en la Endoscopia Digestiva
}

\author{
D. LEDRO CANO
}

\section{Servicio de Medicina Interna. Sección de Aparato Digestivo. Complejo Hospitalario} de Jaén

\author{
TRAINING SIMULATORS IN DIGESTIVE ENDOSCOPY
}

\begin{abstract}
RESUMEN
Existen importantes áreas en la Medicina clínica como las técnicas avanzadas, el cuidado óptimo al paciente y la garantía de calidad. El incremento de técnicas mínimamente invasivas en los campos de la Gastroenterología y de la Cirugía, así como los efectos de las curvas de aprendizaje sobre la aparición de complicaciones, han suscitado recientemente un debate acerca de los estándares de formación. La alerta social y la creciente presión legal para mostrar y certificar la competencia ha venido a incrementar la importancia de la formación en la Medicina Intervencionista. Aunque la medicina basada en la evidencia rápidamente ha adquirido el estándar de referencia para las modalidades terapéuticas, la responsabilidad de la educación, incluidas las bases teóricas así como la adquisición y el perfeccionamiento de las habilidades manuales en la endoscopia digestiva, es aún una cuestión del propio médico. Las habilidades prácticas se adquieren rutinariamente, al practicar con pacientes, al principio bajo la supervisión de un endoscopista experto. El desarrollo de nuevos simuladores de endoscopia ha suscitado el debate acerca de si la obtención de la formación en habilidades manuales básicas es mejor externamente a los pacientes. Este artículo presenta una panorámica de los simuladores destinados a la formación, actualmente disponibles, así como las cuestiones asociadas con ellos.
\end{abstract}

PALABRAS CLAVE: Endoscopia. Formación. Simuladores.

\begin{abstract}
Advanced techniques, optimal patient care, and quality management are currently important topics in clinical medicine. The increasing numbers of minimally invasive procedures being carried out in gastroenterology and surgery, and the effects of the learning curve on complication rates with various procedures, have given rise a recently debate on training standards. Public awareness and increasing legal pressure to show and document competence have further contributed to the importance of training in interventional medicine. Although evidence-based medicine is rapidly becoming the gold standard for treament modalities, responsibility for education-including the theoretical background, as wells as acquiring and refining manual skills in gastrointestinal endoscopy- is still a matter for the individual physician. Practical skills are routinely acquired by practicing on patients, initially under the supervision of a senior endoscopist. The development of new endoscopy simulators has brought out the debate whether training in basic manual skills is better obtained outside the patient. This paper presents an overview of the training simulators currently available and issues associated with them.
\end{abstract}

KEY WORDS: Endoscopy. Training. Simulators.

Ledro Cano D. Simuladores de formación en la Endoscopia Digestiva. An Med Interna (Madrid) 2004; 21: 456-460.

\section{ESTUDIOS ACERCA DE LA FORMACIÓN} Y GUÍAS DE ACTUACIÓN

Los cirujanos tienen una larga tradición de confeccionar y mantener el manual del residente, donde se incluyen sus técnicas, esta práctica es muy reciente en nuestro país, especialmente en los gastroenterologos. Desde los años ochenta, los residentes son obligados a conservar un diario de las técnicas realizada, al menos en Europa Occidental y Estados Unidos. (1) En España, comenzó a ser introducido tímidamente el libro del residente, a finales de los años noventa, sin carácter obligatorio, si bien en algunos centros hospitalarios docentes, se redactan certificaciones sobre el número de procedimientos de una determinada técnica por el residente. (2) Al mismo tiempo, las sociedades médicas han comenzado a tratar la cuestión del número de procedimientos requeridos, para ser considerado autónomo en una determinada técnica endoscópica. La Federación de Sociedades de Enfermedades Digestiva recomienda un mínimo de 50-100 técnicas como mínimo para considerar la competencia en la endoscopia digestiva alta y en

\footnotetext{
Trabajo aceptado: 8 de junio de 2004
} 
la endoscopia digestiva baja. (3) Wington obtuvo estimaciones sobre el número necesario de procedimientos para alcanzar la competencia en médicos especialistas de medicina interna (4), médicos internos y residentes de medicina interna (5) y gastroenterologos (6).

Los dos primeros grupos opinaron que una media de 25 endoscopias digestivas altas o endoscopias digestivas bajas eran suficientes, mientras los gastroenterologos pensaron que una media de 75 endoscopias digestivas altas y 88 endoscopias digestivas bajas, eran el número necesario de procedimientos. El American Board of Internal Medicine entrevisto a directores de programas de formación en endoscopia y comunicaron que un mínimo de 85 endoscopias digestivas altas y 75 endoscopias digestivas bajas sería lo razonable (7).

Dado que en el caso de las colonoscopias, el colon entero ha de ser examinado, para tener seguridad de que ninguna lesión no ha sido visualizada, la habilidad para alcanzar ciego es el criterio más frecuente para evaluar esta técnica. De hecho, cerca de la mitad de los cánceres, que no se diagnostican, son debidos a exploraciones incompletas (8).

Un estudio prospectivo sobre las colonoscopias en el Reino Unido demostró que la colonoscopia es frecuentemente incompleta y que no alcanza el objetivo de la intubación cecal en al menos el $90 \%$ de los casos y que la formación en colonoscopia es frecuentemente inadecuada (9). Por esta razón, se desarrollan cursos prácticos de colonoscopia, que consisten en una introducción teórica, seguida de la realización de cuatro colonoscopias bajo supervisión, para unificar criterios de formación y educación, dichos cursos están financiados por el Departamento de Salud del Reino Unido (10).

Parry y Williams de Nueva Zelanda conservan archivos acerca de la colonoscopias realizada. Ellos observaron que, incluso con 305 procedimientos, el ciego sólo se alcanzaba en el $91 \%$ de los casos (11).

Cass realizó un estudio piloto que incluía a siete residentes de aparato digestivo y cinco residentes de cirugía general, utilizando un programa informático para la evaluación de su labor, basado en medidas simples de competencia, después de cada procedimiento (12). Sus resultados mostraron que al menos 100 endoscopias digestivas altas y 100 endoscopias digestivas bajas eran necesarias para alcanzar el $90 \%$ de las medidas simples de éxito. En el caso de la colonoscopia, eran pasar la flexura esplénica y alcanzar el ciego y en el caso de la endoscopia digestiva alta, era intubar el esófago y atravesar el píloro. En cada procedimiento, se observo si los residentes reconocían si existía alguna patología y en que consistía.

Marshall siguió la evolución de nueve residentes de gastroenterología y midió su éxito en alcanzar ciego, durante los meses finales de su primer y segundo años (13). Encontró sólo una tasa de éxito del $86 \%$ en alcanzar el ciego, después de que los residentes realizaran 328 procedimientos.

Chak observó la evolución de la realización de colonoscopias por cinco residentes de primer año e incluso residentes de segundo año, durante cuatro meses en un programa de residencia de endoscopia digestiva de dos años. Tras 123 colonoscopias, comunico que sólo en el $63.7 \%$ de los procedimientos se alcanzaba ciego (14).

Church evaluó a varios residentes de Cirugía General en sus primeros 125 procedimientos, constatando que en las últimas 25 colonoscopias, el ciego se alcanzo sólo en el $72 \%$ de los casos (15).
A la vez que la información sobre gastroenterologos y cirujanos crecía, los médicos de familia empezaron a recopilar sus resultados. Godreau (16), Rodney (17) y Hopper (18) comunicaron sus experiencias en la práctica de las colonoscopias, después de formación en cursos, estancias cortas o en el propio quehacer diario. Ellos comunicaron tasas de éxito de alcanzar ciego de 83, 52 y 75\% en 157,100 y 1.048 procedimientos, respectivamente.

Desagraciadamente, la gran serie comunicada por Hopper no fue separada por número de procedimientos previos, sino por el uso de sedación y por el sedante utilizado.

Tassios evaluó a ocho residentes de gastroenterología y aplicando una análisis de regresión, estimó que la tasa de éxito en alcanzar ciego era del $67 \%$ tras 100 colonoscopias y del $77 \%$ tras 180 (19).

La mayoría de estos estudios incluían un número escaso de pacientes. En un intento de recopilar el máximo de información posible. Cass, una vez mas, realizó un estudio que incluía 14 centros con 135 residentes de Aparato Digestivo durante una residencia de tres años. Los datos preliminares fueron presentados en 1996. El estudio concluyo que, al menos 130 endoscopias digestivas altas y 180 endoscopias digestivas bajas han de ser realizadas para alcanzar todos los criterios de competencia en más del 90\% de los casos (20).

Al ver la luz, estos datos, las sociedades de gastroenterología han comenzado a incrementar el número de procedimientos que recomiendan para alcanzar la competencia. Algunas de estas recomendaciones están reflejadas en la tabla I.

TABLA I

RECOMENDACIONES ACERCA DEL MÍNIMO NÚMERO DE EXPLORACIONES PARA ALCANZAR LA COMPETENCIA

\begin{tabular}{lccc}
\hline Organización & EDA & EDB & CPRE \\
\hline ASGE & 100 & 100 & 100 \\
BSG & 300 & 100 & 150 \\
AUSTRALIA & 200 & 100 & 200 \\
UEMS & 300 & 100 & 150 \\
\hline
\end{tabular}

EDA: Endoscopia Digestiva Alta; EDB: Endoscopia Digestiva Baja; CPRE: Colangiopancreatografía retrógrada endoscópica; ASGE: Asociación Americana de Endoscopia Digestiva; BSG: Sociedad Británica de Gastroenterología; y UEMS: Unión Europea de Médicos Especialistas.

Es bastante claro que más datos extraídos de la práctica clínica, incluyendo resultados, serían necesarios para poder dar recomendaciones firmes y basadas en la evidencia.

SIMULADORES DE FORMACIÓN. MUÑECOS PLÁSTICOS Y OTROS MODELOS ESTÁTICOS

Los modelos iniciales experimentales para la formación en endoscopia fueron fabricados de plástico. En 1974, Classen y Ruppin en Erlangen, Alemania, presentaron un muñeco de plástico con forma anatómica que permita la exploración del tracto gastrointestinal superior con un panendoscopio flexible. (21) El profesor Williams y su grupo en Londres han estado desarrollando desde los primeros setenta, muñecos semirígidos que permitían a los residentes, la adquisición de habilidades básicas en la colonoscopia flexible (22). 
Modelos estáticos similares han sido desarrollados por Heinkel, de forma hemisférica y por Lucero, para el tracto gastrointestinal inferior, que incluía dibujos de hallazgos patológicos $(23,24)$.

En cursos de desarrollo profesional sobre endoscopia terapéutica en la Universidad de Dundee, Reino Unido, utilizan modelos que nos sirven para simular tratamientos endoscópicos tales como la gastrostomía endoscópica percutánea, la inyección de sustancias esclerosantes, la aplicación de sondas de calor, la ligadura de varices esofágicas con materiales variados, sencillos y baratos como plásticos, gomaespuma y cartón.

De la misma manera en cursos prácticos sobre endoscopia digestiva baja, desarrollados en centros europeos tales como el Royal Liverpool and Broadgreen University Hospital y el Erasmus Medical Center, de Rotterdam, Holanda, son utilizados modelos plásticos del tracto digestivo inferior, como parte de la medidas encaminadas para adquirir las habilidades colonoscópicas.

Actualmente, el modelo más avanzado de simulador estático es el modelo interphant, desarrollado por Grund y su grupo en Tübingen, Alemania. (25) Este modelo tridimensional con forma anatómica y compuesto por látex representa la anatomía del tracto gastrointestinal, el aparato respiratoria y gran parte del páncreas y del árbol biliar. Un sistema modular permite el intercambio entre diferentes situaciones anatómicas y hallazgos patológicos. El muñeco está compuesto, en su mayoría de material no biológico. Los hallazgos patológicos tales como los pólipos, tumores y las estenosis malignas o benignas son creadas mediante un material electroconductor, con consistencia de cera y que puede ser modelado. El material puede ser tratado de la misma manera que el tejido natural, de modo mecánico (toma de biopsia, por ejemplo), ópticamente (varias clases de láser), y eléctricamente ( polipectomía y esfinterotomía).

Tanto cortes mecánicos y eléctricos, ablación, coagulación y otras medidas terapéuticas pueden ser reproducidas muy fieles a la realidad $(26,27)$. Este modelo es utilizado con éxito en las reuniones de la Sociedad Alemana de Endoscopia Digestiva así como en una variedad de cursos distribuidos por toda Alemania tales como en Erlangen y Hildesheim.

Sin embargo, estos modelos plásticos para procedimientos terapéuticos carecen de capas de tejido separadas de una pared gastrointestinal estructurada naturalmente, con capas mucosas y submucosas. Es por tanto más difícil simular tratamientos que incluyen la pared del intestino, especialmente la mucosa, tales como la inyección de sustancias, ligadura con bandas, resección mucosa endoscópica y el tratamiento de la úlcera péptica. Como intento de resolver dichos problemas, el grupo de Grund ha incluido partes de órganos de animales en su modelo.

\section{SIMULADORES INFORMÁTICOS}

En 1990, Williams describió, por primera vez un simulador informático para la colonoscopia y la colangiopancreatografía retrógrada endoscópica (28). En una revisión más reciente del desarrollo de la simulación para el aprendizaje de la colonoscopia, el propio Williams afirma que en 1982, su grupo adaptó un simple videojuego electrónico que permitía la coordinación de ambas manos para ser entrenadas. El siguiente simulador fue conectado a un ordenador personal, con las limitaciones mecánicas del colonoscopio de prueba por los frenos de fricción. Incluso en aquella época, el sistema incluía signos audibles tales como gruñidos y protestas del hipotético paciente si se ejercía una fuerza inapropiada o bien una insuflación exagerada (22).

A mitad de los años ochenta, Noar en Baltimore, comenzó a desarrollar un simulador asistido por ordenador, interesante y realístico, conocido como sistema interactivo y robótico de simulación de endoscopia (RIES) para ERCP y EDA (29). El objetivo era integrar un endoscopio normal en un ambiente interactivo y por tanto crear una apariencia visual de casi realidad. El sistema alcanzó un alto nivel de perfección técnica, con esfínteres funcionales en la papila de Vater y un feedback táctil. Además permitía movimientos de los catéteres, incluso una esfinterotomía virtual era posible (29). Sin embargo, los simuladores informáticos en aquella época requerían grandes computadoras, y por tanto el sistema no tuvo aceptación global $(30,31)$.

Actualmente, debido a los tremendos desarrollos en los ordenadores personales, incluso los sistemas de los mismos son mucho más potentes que las computadoras más avanzadas hace una década.

Este puede ser el motivo del desarrollo de los últimos modelos de simulación endoscópica por ordenador:

1. Simbionix GI mentor. Este simulador en forma de muñeco fue presentado por Bar-Meir en Israel (32), El residente puede elegir entre varios niveles de dificultad para entrenamiento, procedimientos diagnósticos y técnicas terapéuticas virtuales en el tracto digestivo superior, incluyendo páncreas y sistema biliar, así como el tracto digestivo inferior. El sistema crea un ambiente virtual muy cercano a la realidad.

2. Simbionix GI mentor II. La principal diferencia con su predecesor radica en la rapidez en la carga del programa, la pantalla es sensible al tacto y el diseño del muñeco sea simplificado. Ambos simuladores incluyen módulos de hemostasia, endoscopia digestiva alta, endoscopia digestiva baja, ecoendoscopia del tracto superior y colangiopancreatografía retrógrada endoscópica. Estos simuladores son utilizados en diversos cursos prácticos de endoscopia en Estados Unidos, Holanda, Alemania y Reino Unido. Es líder de ventas en Europa.

3. AccuTouch Endoscopy Simulator. La principal diferencia con los simuladores Simbionix es el poderoso feedback, que ejerce el sistema. Es utilizado en numerosos cursos en Reino Unido, Estados Unidos y próximamente en nuestro país, en Granada, en la Fundación IAVANTE, el próximo otoño. Ocupa el liderazgo en ventas en Estados Unidos.

4. La empresa Olympus está desarrollando un nuevo simulador, que probablemente será presentado en la próxima Semana de Enfermedades Digestivas en Nueva Orleans. (DDW2004).

\section{FORMACIÓN ENDOSCÓPICA EN ANIMALES ANESTESIADOS}

Cursos de formación en endoscopia han sido realizados, utilizando perros y cerdos, para endoscopia digestiva alta y baja, así como de manera especial para las técnicas de colangiopancreatografía retrógrada endoscópica, durante más de 10 años $(33,34)$. En nuestro país se han realizado, bajos los auspicios de la asociación española de endoscopia digestiva, en el centro de cirugía de mínima invasión de Cáceres. 
Los cerdos han sido utilizados para el aprendizaje de técnicas básicas de CPRE desde los primeros años noventa. Las principales limitaciones de este modelo de entrenamiento son los aspectos éticos, las consideraciones sobre los derechos de los animales, los problemas higiénicos, la necesidad de endoscopios especiales y los altos costes del equipamiento y del personal. Además, los procedimientos han de realizarse en laboratorios de animales, que requieren permisos diferentes de los de los experimentos en animales, así como la asistencia de veterinarios y anestesistas. Las ventajas son la sensación de tejido natural, elasticidad y feedback táctil de estructuras orgánicas, similares a las humanas. La anatomía del perro es complicada por el pequeño tamaño de la segunda porción duodenal. La anatomía del cerdo es relativamente parecida a la humana, aunque existen diferencias tales como la existencia de dos conductos independientes que drenan a duodeno, por dos orificios separados: uno biliar y otro pancreático. La papila biliar está situada a $2 \mathrm{~cm}$. del píloro, en el techo del bulbo duodenal. La papila pancreática es difícil de encontrar por su pequeño tamaño y su localización profunda en el duodeno. Además, una estructura polipoidea, conocida como el torus pilórico pudiera imitar un cálculo enclavado en papila, y podría permitir el aprendizaje de técnicas de precorte. Algunos problemas de explorar el tracto digestivo superior del cerdo son la ectasia gástrica y la gran distancia al píloro.

\section{SIMULADORES DE PARTES DE ANIMALES}

Los modelos de biosimulación consisten principalmente de simuladores que usan órganos de animales, obtenidos usualmente recién sacrificados en las carnicerías. Este tipo de simulador es utilizado comúnmente en las ciencias quirúrgicas tales como la cirugía laparoscópica tanto torácica como abdominal, ginecología, urología, orl y traumatología.

\section{Bibliografía}

1. American Society for Gastrointestinal Endoscopy. Trainee evaluation form. Manchester, Massachusetts: American Society for Gastrointestinal Endoscopy, 1982.

2. http://www.diariomedico.com/profesion/prof270398com.html.

3. Federation of Digestive Disease Societies. Guidelines for Training in endoscopy. Manchester, Massachusetts: Federation of Digestive Disease Societies, 1981.

4. Wington RS, Nicolas JOA, Blank LL. Procedural skills of the general internist: a survey of 25000 physicians. Ann Intern Med 1989; 111: 1023-34.

5. Wington RS, Blank LL, Nicolas JOA, Tape TG. Procedural skills training in internal medicine residencies. Ann Intern Med 1989; 111: 932-8.

6. Wington RS, Blank LL, Monsour H, Nicolas JOA. Procedural skills of practicing gastroenterlogists: a national survey of 700 members of the American College of Physicians. Ann Intern Med 1990; 113: 540-6.

7. American Board of Internal Medicine. Results of procedure survey of gastroenterology program directors. Am Intern Med Newsletter,Spring/Summer 1990: 4-5.

8. Haseman JH, Lemmel GT, Rahmani EY, Rex DK. Failure of colonoscopy to detect colorectal cancer. Gastrointest Endosc 1997; 45: 451-5.

9. Bowles CJ, Leicester R, Romaya C, Swarbrick E, Williams CB, Epstein O. A prospective study of colonoscopy practice in the UK today: are we adequately prepared for national cancer screening tomorrow.? Gut 2004;
Freys comunico en 1995, sobre el uso de un estomago de cerdo para enseñar endoscopia diagnóstica (35). Ellos fijaron un estomago limpio de cerdo con agujas sobre un tablón de corcho e introdujeron un endoscopio, con el objeto de enseñar endoscopia digestiva alta. En 1996, el grupo del Prof.Hahn desarrollaron un nuevo enfoque al aprendizaje y a la enseñanza de la endoscopia digestiva superior terapéutica, utilizando paquetes de órganos del tracto digestivo superior del cerdo, especialmente preparados. Una amplia variedad de técnicas terapéuticas pueden ser practicadas en el tracto digestivo superior, en el sistema biliar y en el páncreas $(36,37,38)$.

Inicialmente, fue utilizado el modelo de biosimulación de Neumann, que consistía en un muñeco con una forma anatómica. Al fondo de la estructura, los órganos viscerales del cerdo eran utilizados para técnicas quirúrgicas tales convencionales como laparoscópicas. Se incluyo una bomba, para perfundir las arterias de los órganos para procedimientos laparoscópicos tales como la colecistectomía. Basados en este idea, Hochberger simuló el sangrado a chorro en el estomago, al perforar la pared del órgano con una cánula de plástico, conectada a un sistema de perfusión pulsátil. Se realizaron modificaciones, para permitir el paso del gastroscopio terapéutico y el duodenoscopio, permitiendo, simular múltiples intervenciones terapéuticas. Posteriormente la cánula fue sustituida por un arteria del cerdo, cosida a la pared anterior gástrica. Las varices fueron reproducidas mediante la inyección submucosa de colorante salino. Nuevas patologías susceptibles de tratamiento endoscópico, fueron introducidas en el modelo, como pólipos, mediante suturas tipo bolsa de tabaco y estenosis, producidas mediante compresión extrínseca externa. También fueron integrados el sistema hepatobiliar, para el aprendizaje de CPRE.

Mini-EASIE y EASIE compacto son simuladores simplificados, que incluyen los mínimos requerimientos para la realización de endoscopia terapéutica. Un estudio que compara el EASIE original y los modelos simplificados, no muestra diferencias significativas, en cuanto a efectividad (39).

53 (2): 277-83.

10. http://www.rcseng.ac.uk/surgical/gastro

11. Parry BA, Williams SM. Competency and the colonoscopist: a learning curve. Aust NZ J Surg 1991;61:419-22.

12. Cass OW, Freeman ML, Peine CJ, Zera RT, Onstad GR. Objective Evaluation of endoscopy skills during training. Ann Intern Med 1993; 118: 40-4.

13. Marshall JB. Technical proficiency of trainees performing colonoscopy: a learning curve. Gastrointest Endosc 1995;42:287-91.

14. Chak A, Cooper GS, Blades EW, Canto M, Sivak MV Jr. Prospective assessment of colonoscopic intubation skills in trainees. Gastrointest Endosc 1996; 44: 54-57.

15. Church JN. Learning colonoscopy: the need for patience (patients). Am J Gastroenterol 1993; 88: 1569

16. Godreau CJ. Office-based colonoscopy in family practice . Fam Pract Res J 2001; 12: 313-20.

17. Rodney WM, Dabov G, Cronin C. Evolving colonoscopy skills in a rural family practice: the first 293 cases. Fam Pract Res J 1993; 13: 43-52.

18. Hopper W, Kyler KA, Rodney WM. Colonoscopy by a family physician: a 9-year experience of 1048 procedures. J Fam Pract 1996; 43 561-6.

19. Tassios PS, Ladas SD, Grammenos I, Demertzis K, Raptis SA. Acquisition of competence in colonoscopy: the learning curve of trainees. Endoscopy 1999; 31: 702-6. 
20. Cass OW. Acquisition of competency in endoscopic skills during training: a multicenter study. Gastrointest Endosc 1996; 43: 308.

21. Classen M, Ruppin H. Practical training using a new gastrointestinal phantom. Endoscopy 1974; 6: 127-31.

22. Williams CB, Saunders BP, Bladen JS. Development of colonoscopy teaching simulation. Endoscopy 2000; 32: 901-5.

23. Heinkel H, Kimming M. Phatomtraining zur Ausbildung der endoskopischen Magenuntersuchung. Z Gastroenterol 1972;10: 393-8.

24. Rey JF, Romanczyk T. The development of experimental models in the teaching of endoscopy: an overview. Endoscopy 1995; 27: 101-5.

25. Grund KE, Bräutigam D, Zindel C, Lothert M, Farin G. Interventionsfähiges Tübinger Simulationsmodell Interphant für die flexible Endoskopie. Endosk Heute 1998; 11: 134.

26. Grund KE, Straub T, Farin G. Integriertes Ausbildungskonzept für die diagnostische und interventionelle flexible Endoskopie. Z Gastroenterol 1999; 37: 943.

27. Grund KE, Zindel C, Bräutigam D, Köhn P, Farin G. "Künstliches Gewebe" zum Training von Interventionen in der Endoskopie. Endosk heute 1997; 10: 177.

28. Williams CB, Baillie J, Gillies DF, Borislow D, Cotton PB. Teaching gastrointestinal endoscopy by computer simulation: a prototype for colonoscopy and ERCP. Gastrointest Endosc 1990; 36: 49-54.

29. Noar MD. Robotics Interactive endoscopy simulation of ERCP/sphincterotomy and EGD. Endoscopy 1992; 24 (Suppl. 2): 539-41.

30. Noar MD, Soehendra N. Endoscopy simulation training devices. Endoscopy 1992; 24: 159-66.
31. Beer-Gabel M, Delmotte S, Muntak L. Computer assisted training in endoscopy (C.A.T.E.): from a simulator to a learning station. Endoscopy 1992; 24 (Suppl. 2): 534-8.

32. Bar-Meir S. A new endoscopic simulator. Endoscopy 2000; 32: 898-900.

33. Gholson CF, Provenza JM, Silver RC, Bacon BR. endoscopic retrograde cholangiography in the swine: a new model for endoscopic training and hepatobiliary researcgh. Gastrointest Endosc 1990; 36: 600-3.

34. Noar MD. An established porcine model for animate training in diagnostic and therapeutic ERCP. Endoscopy 1995; 27: 77-80.

35. Frey SM, Heimbucher J, Fuchs KH. Teaching upper gastrointestinal endoscopy: the pig stomach. Endoscopy 1995; 27: 73-6.

36. Hochberger J, Neumann M, Maiss J, Bayer J, Nägel A, Hahn EG Erlanger Ausbildungs-Simulator für die interventionelle Endoskopie (EASIE). Eine neue Perspektive für die qualitätsorientierte praktische Ausbuilding. Endosk Heute 1998; 4: 23-5.

37. Neumann M, Mayer G, Ell C, Felzmann T, Reingruber B, Horbach T et al.The Erlangen Endo-Trainer: life-like simulation for diagnostic and interventional endoscopic retrograde cholangiography. Endoscopy 2000; 32: 906-10

38. Hochberger J, Neumann M, Maiss J, Hohenberger W, Hahn EG. EASIE (Erlangen Active Simulator for Interventional Endoscopy), a new biosimulation model: first experiences gained in training workshops (abstract). Gastrointest Endosc 1998; 47 (Suppl.): AB116.

39. Maiss J, Hildebrand V, Bayer J, Hahn EG, Hochberger J. Mini-EASIE: ein neues, auf die Belange der interventionellen Endoskopie reduziertes Trainingsmodell. Endosk Heute 1999; 12: 53. 